\title{
Ciclo de Mejora en el Aula e Implantación de la Inteligencia Emocional en la Asignatura "Fisioterapia en Atención Temprana"
}

\section{The improvement cicle in Classroom and Emocional Intelligence in the Subject "Phisical Therapy in Early Chilhood Intervention"}

MARÍA LUISA BENÍTEZ LUGO

ORCID:https://orcid.org/0000-0003-2058-8799

Universidad de Sevilla,

Facultad de Enfermería, Fisioterapia y Podología

Departamento de Fisioterapia

Correo electrónico: marisabeni@us.es

Fecha de recepción: 15-06-19

Fecha de aceptación: 26-06-19

DOI: http://dx.doi.org/10.12795/9788447221912.025

Pp.: 577-600 


\section{Resumen:}

El Ciclo de Mejora en el Aula (CIMA) se desarrolla sobre una asignatura optativa del segundo curso del Grado en Fisioterapia. Tanto las actividades teóricas como prácticas se someten a procesos innovadores, incluyendo de manera transversal la implantación de la Inteligencia Emocional (IE) en el aula. La especialidad sobre la que versa esta materia comprende la intervención sobre niños, familia y entorno. En este sentido se ha visto pertinente formar al alumnado en la adquisición de las competencias transversales consideradas como poderosas, reconocidas como "power skills". Para evaluar el proceso utilizamos un cuestionario de ideas iniciales, asi como dos cuestionarios específicos para evaluar el nivel previo de IE (clima de clase y evaluación de las competencias emocionales). Durante todo el curso académico se diseñan diversas dinámicas, las cuáles se acompañan de una reflexión posterior al aprendizaje por descubrimiento, así como todo un abanico de actividades que han ido ayudando al alumnado a superar sus limitaciones en el proceso de aprendizaje. Además hemos realizado diferentes rúbricas para evaluar las actividades desarrolladas desde la metodología de "Aprendizaje-Servicio". Del mismo modo, la profesora ha sido evaluada en cada intervención a través del diario reflexivo del docente así como con una encuesta final de resultados. El análisis de los resultados en las escaleras evidencia que, en todos los casos, el alumnado ha subido de nivel. Por otro lado, el alumnado resalta como actividades de aprendizaje más significativo las siguientes: ApS en Escuelas Infantiles (64,28\%), dinámicas en IE (35,7\%), role-playing de familias y despedida final $(21,42 \%)$ y presentación de la asignatura, murales creativos y diario de clase (7,14\%). En todos los casos la satisfacción del alumnado con la asignatura y con la docente es excelente.

Palabras claves: Fisioterapia en Atención Temprana, Grado en Fisioterapia, Docencia Universitaria, Experimentación Docente Universitaria, Inteligencia Emocional. 


\section{Abstract:}

The improvement cycle in Classroom (ICIS) is development about an optional subject of the Physiotherapy Degree. Theoretical and practical activities are subjected to innovative processes, including the implementation of emotional intelligence in the classroom. The specialty on this subject includes the treatment about children, families and environment, so it has been pertinent to train the students to get power skills. This process had been evaluated with an initial questionnaire and others two-specific questionnaires to test the previous level of emotional intelligence (class climate and emotional skills). We are based in ICIS which had been applied for years ago. We have also made different rubrics to evaluate several learning activities that had been development with "Learning-Service" methodology. The teacher and all activities had been evaluated through the teacher's reflective diary and final questionnaire. The analysis of the results shows that, in all cases, the students have increased the learning level. On the other hand, the students highlighted as the most significant learning activities the following: LS in Children's Schools (64.28\%), Dynamics in Emotional Intelligence (35.7\%), Family Role-Playing and Final Class (21.42 $\%)$ and Presentation of the subject, Creative Murals and class diary (7.14\%). In all cases, the satisfaction of the students with the subject and with the teacher is excelent.

Key words: "Physical Therapy in Early Chilhood Intervention", "Physiotherapy Degree", “University Teaching","University Teaching Experimentation" and "Emocional Intelligence"

Jornadas de Formación e Innovación Docente del Profesorado | № 2 (2019) Esta obra se distribuye con la licencia Creative Commons 


\section{Breve descripción del contexto:}

La materia sobre la que se ha realizado el CIMA, dentro del Programa de Formación e Innovación Docente del Profesorado, es optativa y está incluida dentro del segundo curso y segundo cuatrimestre de la titulación. Esta asignatura, denominada "Fisioterapia en Atención Temprana" (FAT), tiene un total de 6 créditos distribuidos en 45 horas teóricas y 15 horas prácticas. El número total del alumnado es 21. Las horas teóricas son impartidas en grupo único y las clases prácticas se distribuyen en tres subgrupos.

Esta asignatura ya ha tenido CIMAs previos (presentados en la convocatoria anterior de la REFID), por esta razón, en esta convocatoria se pretende perfeccionar las actividades propuestas en el curso pasado y modificar y reflexionar sobre aquellos contenidos que no fueron mejorados entonces. Asimismo, como futuros profesionales de la salud y como alumnado de una asignatura que aborda la diversidad funcional, resulta interesante añadir de manera transversal, contenidos y actividades sobre IE. De esta manera la elaboración del CIMA en este curso, se realizó sobre la totalidad de la asignatura.

\section{Diseño previo del Ciclo de Mejora en el Aula:}

\section{Mapa de contenidos, problemas y núcleos de trabajo en el aula:}

En este curso académico se realiza el ciclo de mejora en el aula sobre la asignatura completa. En la figura 1, se presenta la red de contenidos que da respuesta a todas las preguntas sugeridas en el aula y que engranan todas las competencias (conceptuales, procedimentales y actitudinales) que se tejen a través de esta asignatura y todas las secuencias de actividades. Aunque cada una de las unidades tiene su mapa conceptual específico, esta figura muestra la red global donde cada pregunta y competencia aparecen a modo de conexiones neurales. 
MARÍA LUISA Benítez LUGo

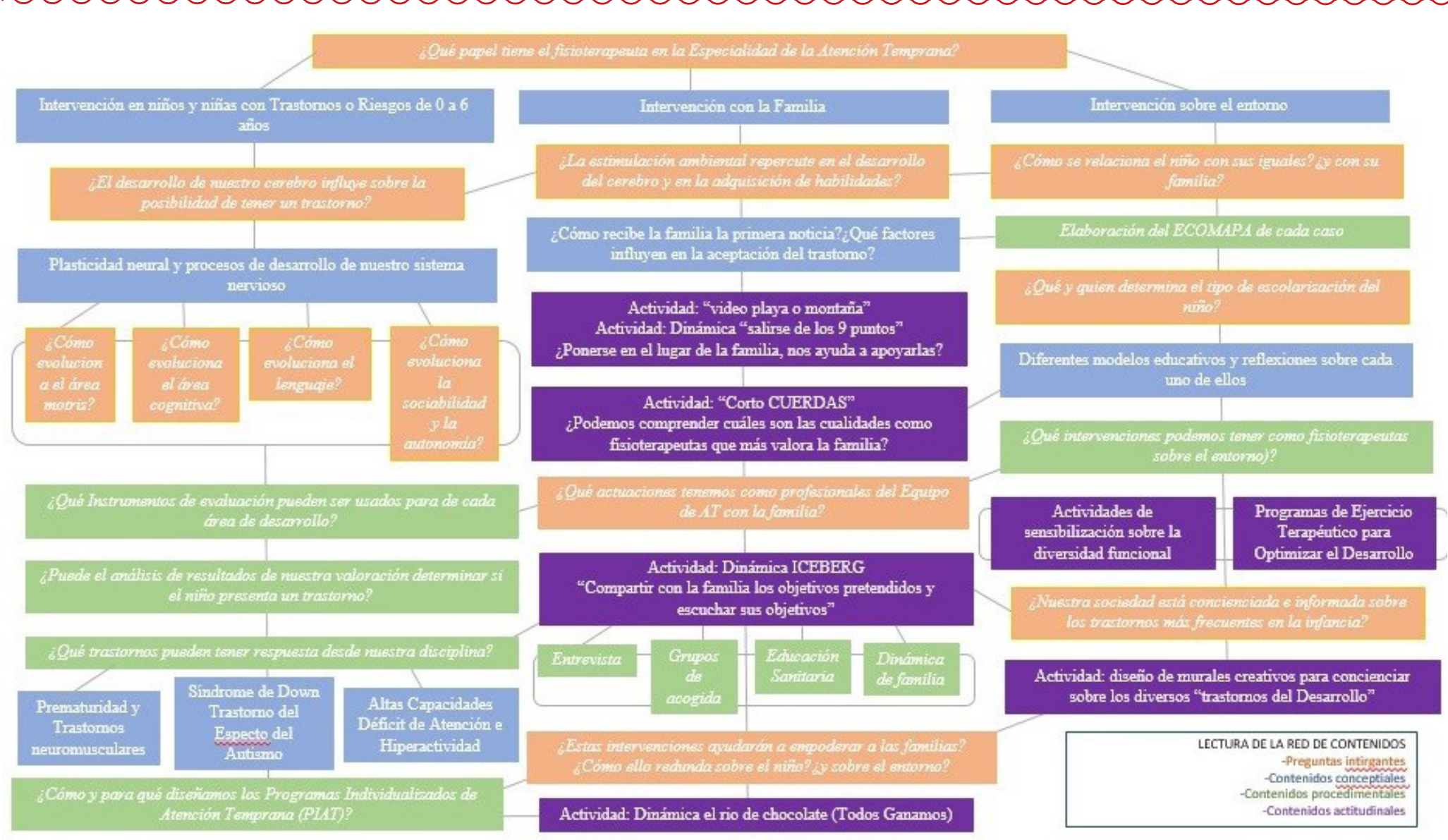

Figura 1. Red de contenidos y preguntas de la asignatura FAT

Jornadas de Formación e Innovación Docente del Profesorado | № 2 (2019) Esta obra se distribuye con la licencia Creative Commons

Reconocimiento-NoComercial-SinObraDerivada

Internacional (CC BY-NC-ND 4.0.) 


\section{María LUISA Benítez LUgo}

Por otro lado, cada uno de los contenidos se desmenuza en más preguntas intrigantes y en más competencias, conformando todo el tejido de conocimiento que corresponde a esta rama del conocimiento. Un ejemplo de ello es la figura 2, donde presentamos una red de contenidos y preguntas de un bloque específico, ampliado de la red general.

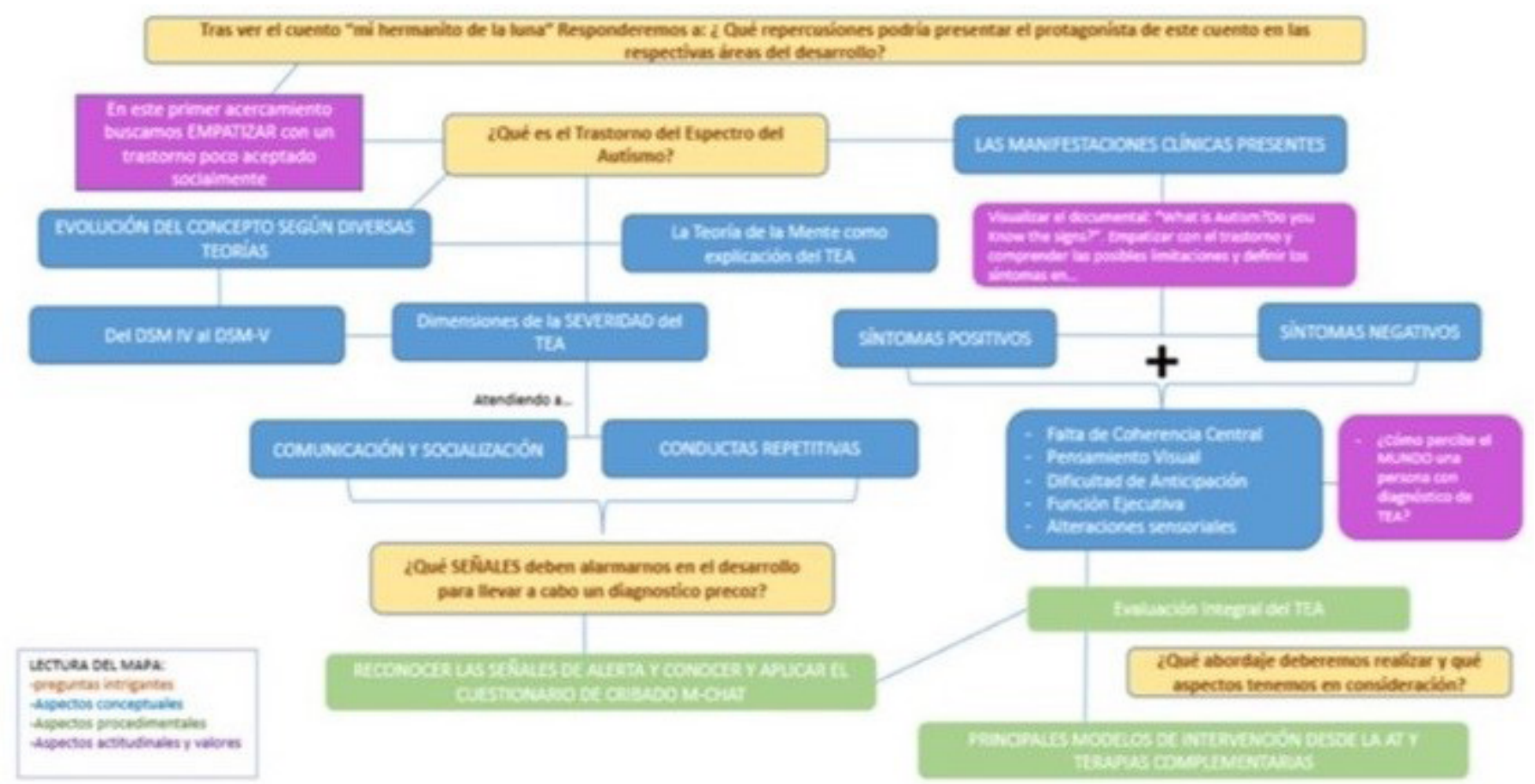

Figura 2. Red de contenidos y preguntas del tema "Abordaje del Trastorno del Espectro del Autismo desde la Fisioterapia"

Jornadas de Formación e Innovación Docente del Profesorado I № 2 (2019) 
En todo el ciclo tenemos como premisa ir respondiendo a los contenidos que nos planteamos de manera tridimensional, ayudando al alumnado a adquirir los conocimientos, los procedimientos y las competencias que le hacen desarrollarse como profesionales y seres propositivos. Para el diseño de las redes de contenido, así como para el establecimiento del modelo metodológico posible, nos hemos basado en el libro de Porlán y colaboradores (2017).

\section{Modelo metodológico posible y secuencia de actividades:}

El modelo diseñado comienza por una fase de Tiempo de los Estudiantes, donde a través de una pregunta intrigante, trazamos el hilo conductor de la sesión. Posteriormente podemos establecer una segunda fase de teoría y práctica, o bien pasar directamente a la fase de discusión-debate y de este segundo escalón ir a la de teoría-práctica. El sentido de las flechas dependerá de la temática y actividades diseñadas. La última, fase de repaso, permitirá reflexionar y concluir sobre la pregunta conductora de la sesión, repasando los aspectos más esenciales. De manera programada también incluiremos, en función a la temática, las respectivas dimensiones que conforman la IE. Como se observa en la figura 3, a veces podemos pasar directamente a la fase de discusión-debate sin entrar en la teoría. Esto es posible con temas que pueden sustentarse sobre contenidos anteriores o sobre conocimientos que se pueden tener con respecto a lo impartido en otras asignaturas del Grado en Fisioterapia. 
TE: Tiempo de los estudiantes (comenzando siempre por la bienvenida y la pregunta intrigante)

T-P: Contenidos teóricos impartidos alternando la lección magistral con otras actividades más prácticas

I.E: Implementación de la Inteligencia Emocional a través de actividades que fomenten el autodescubrimiento del alumnado y el refuerzo de las diferentes distinciones

D-C: Discusión-Debate a través de hojas de trabajo o de casos clínicos o de experiencia contada

R: Repaso final a través de preguntas reflexivas finales, de cuestionarios Kahoot o de juegos realizados a través de la aplicación Quizlet

Figura 3. Modelo metodológico de la asignatura FAT.

En la tabla 1 se expone la secuencia de actividades desarrolladas durante el CIMA en la asignatura FAT. Para una mejor comprensión, las actividades se clasifican atendiendo a si forman parte de los contenidos teóricos, prácticos o a la implementación de la IE. Del mismo modo, se expone la pregunta a responder y cómo se contesta a través de las respectivas fases del modelo metodológico. En el esbozo de actividades hemos tenido en cuenta las indicaciones de Bain (2004) y Finkel (2008), así como el CIMA realizado el curso anterior por Benítez (2018) y Piñero (2018). Asimismo, para la implantación de la IE hemos recibido los consejos y la reflexión del profesor Ortega-Cámara (2017) y Porlán y colaboradores (2017).

Jornadas de Formación e Innovación Docente del Profesorado | № 2 (2019) Esta obra se distribuye con la licencia Creative Commons Reconocimiento-NoComercial-SinObraDerivada 
Tabla 1. Secuencia de actividades del CIMA en la asignatura FAT (Modelo Metodológico)

PREGUNTA

INTRIGANTE

¿Qué conoci-

mientos previos

posee el estu-

diante sobre la

FAT y la IE?

¿Por quée es

importante el

trabajo con la

Familia desde la

FAT?

\section{ACTIVIDAD EN TEORIA}

-Cuestionario de ideas iniciales

-Análisis de casos clínicos reales Análisis de video “¿Bailamos? Para reflexionar sobre la importancia de la motivación en los niños.

- Kahoot final para analizar los contenidos aprendidos y su grado de entendimiento

:"Playa o Montaña"

- Corto "CUERDAS"

So-

re
respectivas dijadas en grupo

- Desarrollo de sesiones de role-playing con la escenificación de las

\section{-Evaluación y} reflexión sobre instrumentos de evaluación global: Escala Brunet-Lezine R y Escala de Merril-Palmer-R.

-Evaluación de aspectos motrices y planteamiento de
-Distinción conducta Vs Identidad: ¿cómo vemos al niño con trastorno establecido? ¿cómo lo ven sus progenitores? Imagen y reflexión del ICEBERG

Dinámica: "los 9 puntos" objetivos -Evaluación de objetivos cognitivos y planteamiento de actividades -Exposición y análisis de videos reales 
-Análisis de casos clínicos reales - Juego de dibujos abstractos

-Video de la experiencia de la Tortuga Carlota

-Cuento: "Por cuatro esquinitas de nada" para reflexionar sobre la inclusión en la escuela y nuestro papel

-Kahoot final para analizar los contenidos aprendidos y su grado de entendimiento

Documental sobre manifestaciones y desarrollo de mural creativo

-Video sobre cómo siente el entorno un niño con TEA -Video sobre terapias alternativas en TEA

-Video inicial concienciación sobre TDAH

- Video de casos y trabajo de atención Intervención en AACC

-Kahoot final para analizar los contenidos aprendidos y su grado de entendimiento
-Evaluación de las funciones que influyen en el lenguaje -Explicación y desarrollo de la terapia miofuncional - Dinámica y role-playing sobre "Curso de masaje infantil para padres y madres"

-Adaptación de diferentes juegos a diversas situaciones de diversidad funcional (motriz, cognitiva, social, lenguaje)

-Planteamiento, desarrollo y creación de las tareas para sensibilizar y optimizar el desarrollo en edades de 2 a 3 años
-Distinción: Ser mi palabra Vs Rigidez

"Establecimiento de acuerdo y ejemplo de cómo nos sentimos usando el símil de botella llena Vs botella vacía.

-Distinción: "Responsable Vs Víctima". Dinámica: "la venda". Reflexión: “¿Quién es responsable de que tengamos la venda puesta?
-Distinción: Ganar-Ganar Vs Ganar-Perder. Dinámica: "el río de chocolate"

-Distinción: Apoyo Vs ayuda. Dinámica: "el juego de los palillos Reflexiones y aprendizaje sobre todos estos contenidos relacionándolos con los diferentes trastornos abordados. 


\begin{tabular}{|c|c|c|}
\hline $\begin{array}{l}\text { - Cuestionario de } \\
\text { ideas finales } \\
\text {-Cuestionario de } \\
\text { clima de clase final } \\
\text {-Cuestionario de } \\
\text { competencias emo- } \\
\text { cionales finales }\end{array}$ & $\begin{array}{l}\text { - Activida- } \\
\text { des basadas } \\
\text { en Metodolo- } \\
\text { gía Aps desa- } \\
\text { rrolladas en } \\
\text { la Escuela In- } \\
\text { fantil “Nido de } \\
\text { los Perdigo- } \\
\text { nes". Puesta } \\
\text { en escena de } \\
\text { los programas } \\
\text { diseñados y } \\
\text { atrezos. } \\
\text {-Caso clínico } \\
\text { final }\end{array}$ & $\begin{array}{l}\text {-Distinción: Empa- } \\
\text { tía Vs emoción Vs } \\
\text { sentimiento. } \\
\text { Dinámica: "la línea de } \\
\text { abrazos" } \\
\text {-Desarrollo de mu- } \\
\text { ral creativo final } \\
\text { como despedida de la } \\
\text { asignatura }\end{array}$ \\
\hline
\end{tabular}

\section{Cuestionario inicial y final para la evaluación del proceso de enseñanza-aprendizaje:}

Para analizar las ideas previas de los estudiantes, se diseñó un cuestionario de ideas iniciales, utilizando para ello la aplicación de Google Drive, así como las recomendaciones encontradas en Porlán y colaboradores (2017). Del mismo modo, se diseñó un cuestionario de ideas finales para poder analizar, una vez finalizada la asignatura, el grado de aprendizaje de cada alumnado, así como los niveles obtenidos en las escaleras diseñadas en cada bloque de contenidos. Ambos cuestionarios contemplaban las mismas preguntas claves de la materia. Los resultados de estos cuestionarios se detallan en el apartado de evaluación del CIMA en la asignatura FAT. Además utilizamos tanto un cuestionario sobre el clima de clase como la herramienta para analizar las dimensiones emocionales del alumnado en sus estados iniciales y finales (Ugarriza, 2005). También diseñamos rúbricas específicas para el seguimiento continuo en clase, para la actividad de role-playing sobre la familia y para la actividad de ApS desarrollada en la Escuela Infantil, instrumentos que serán utilizados tanto por el alumnado como por la docente (revisión por pares). 


\section{Aplicación del Ciclo de mejora en el Aula:}

\section{Relato resumido de las sesiones}

La utilización del diario del profesor ha sido una herramienta que nos ha permitido ir registrando y analizando de manera reflexiva cada una de las sesiones compartidas con el alumnado. Por otro lado, poner en marcha dos diarios de clase (uno realizado por el alumnado y otro por el profesor), nos ha permitido contrastar nuestras anotaciones con la visión de grupo y usar esa información para ir mejorando cada día. Iniciar la primera clase con la técnica de presentación de la flor, ser un número de alumnado reducido (por ser asignatura optativa), fomentar un clima de cercanía con las comidas en el aula (Bain 2004), así como la inclusión de dinámicas para trabajar de manera transversal la IE, han ayudado a que todas las actividades innovadoras que se han ido desarrollando durante el curso académico, hayan tenido una buena acogida (Finkel, 2008). De todas ellas el alumnado ha mostrado un especial interés por los videos de ejemplos reales que se exponían en clase (por ejemplo intervención directa sobre un niño con un trastorno) para hacerles entender todas las preguntas intrigantes con las que íbamos hilando el aprendizaje, las actividades de trabajo cooperativo (tanto con interacción en contextos reales como en simulados) así como todas las actividades vivenciales y creativas que les han permitido desarrollarse de manera global. En este sentido, los programas realizados en la Escuela Infantil, las dinámicas de familia y las actividades en IE son las que han resultado más relevantes de nuestra experiencia docente. Otra sesión resaltada como significativa ha sido la creación de una exposición en la Facultad para concienciar en materia de diversidad. Para ello el alumnado tuvo que buscar información relevante sobre el trastorno del espectro del autismo y entrevistar a tres personas ajenas al sector 
sanitario y educativo, con el sentido de encontrar los mitos que la sociedad tiene en relación a este trastorno y con la intención de plasmar la realidad sobre murales creativos. De igual manera, hemos utilizado recursos (kahoot y Quizlet) que nos han permitido amenizar las sesiones. En la figura inferior se ejemplifican alguna de las actividades desarrolladas durante esta experiencia, donde se puede observar el transcurrir de las sesiones diarias y el aprendizaje adquirido en cada una de ellas, así como las emociones despertadas en el alumnado y en la docente.
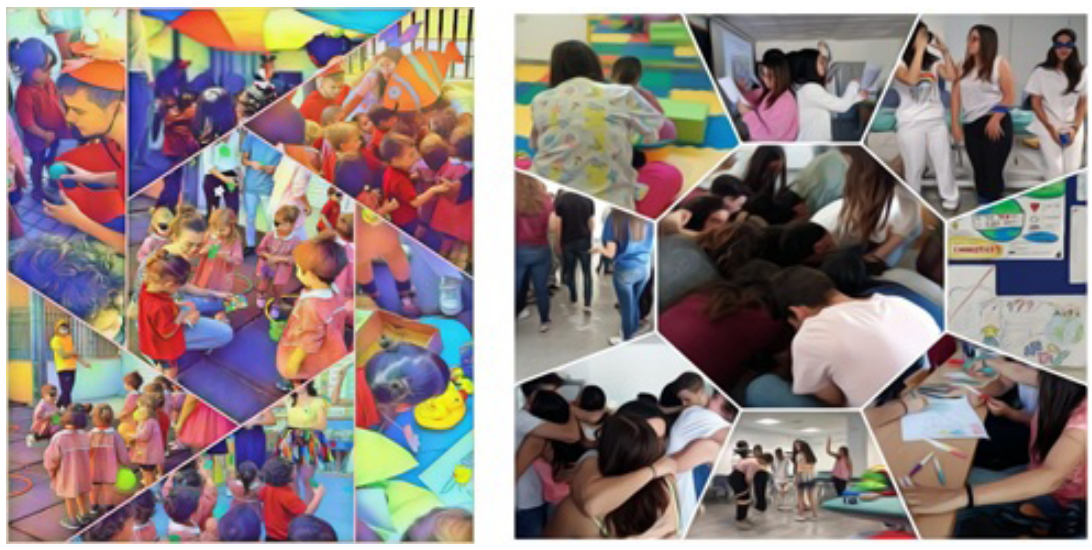

Imagen 1. Ejemplo de las actividades y dinámicas desarrolladas en el aula

No obstante, aquellas tareas que han precisado más profundidad de aprendizaje y más esfuerzo por parte del alumnado, por la alta gama de competencias que se trabajan en ellas (actividad de optimización del desarrollo y sensibilización de la diversidad Infantil en Escuela Infantil "Nido de los Perdigones"), son las que ellos claramente destacan como más fructíferas, argumentando los valores positivos que tiene la metodología de Aprendizaje-Servicio, así como las metodologías creativas. Para que ellos supieran los requisitos que iban a ser evaluables en cada tarea se diseñaron, como se ha dicho, rúbricas específcas que fueron elaboradas con las aportaciones del alumnado y fueron mostradas previamente al desarrollo de la actividad. 


\section{Evaluación del aprendizaje de los estudiantes. Escaleras de aprendizaje}

Para poder realizar una evaluación específica del proceso de aprendizaje, se diseñó un cuestionario inicial y final, del cual extraemos tres de las preguntas más significativas para la asignatura. El número del alumnado que respondió inicialmente al cuestionario es de 13 y en las ideas finales de 14, pero emparejando los cuestionarios por alumno nos quedamos con una muestra final de diez. Esta herramienta se ha realizado en el horario de teoría, donde la asistencia por cuestiones de no obligatoriedad y de la hora no llega nunca al $100 \%$ de la clase. Se presentan los resultados de aprendizaje comparando los niveles de respuesta iniciales y finales, que a su vez han sido categorizados según su grado de complejidad. En la tabla 2 mostramos los niveles de aprendizaje para la primera de las preguntas y en el gráfico 1 se pueden comparar las respuestas, entre el cuestionario inicial y final, a la pregunta ¿Qué es la Atención Temprana y qué papel tiene la Fisioterapia?.

Tabla 2. Posibles niveles de respuesta en la pregunta ¿Qué es la Atención Temprana y qué papel tiene la Fisioterapia?

A. En la definición se incluye intervención sobre niño con trastornos o con riesgo a tenerlo, de 0 a 6 años, sobre la familia y el entorno, dónde se precisa de equipo interdisciplinar

B. Incluye al niño de 0 a 6 años pero olvida a una de las otras dos dimensiones esenciales

C. Incluye solo al niño con trastorno o riesgo a tenerlo pero no considera ninguna de las otras dos dimensiones ni al equipo

D. Solo recoge intervenciones en la infancia sin enmarcar en la edad ni tener en cuenta el resto de dimensiones ni equipo

Jornadas de Formación e Innovación Docente del Profesorado | № 2 (2019) Esta obra se distribuye con la licencia Creative Commons Reconocimiento-NoComercial-SinObraDerivada 


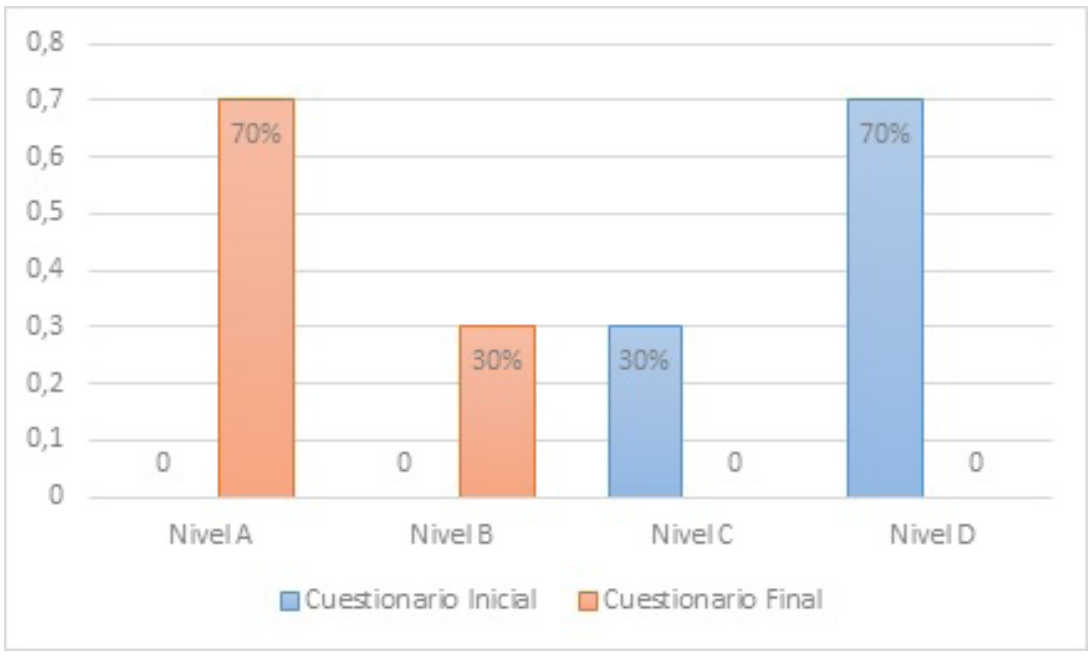

Imagen 1. Ejemplo de las actividades y dinámicas desarrolladas en el aula

Del mismo modo, presentamos la tabla con los niveles de complejidad para la pregunta ¿Crees que se pueden entrenar nuestras capacidades? (tabla 3) y el gráfico 2 que expresa de manera visual dichos resultados.

Tabla 3. Posibles niveles de respuesta a la pregunta ¿Se pueden entrenar nuestras capacidades? ¿A través de qué fenómeno se justifica?

A. Responde de manera justificada a la pregunta explicando en qué consiste el fenómeno de plasticidad neural y generalizándolo en el contexto de trabajo desde la atención temprana

B. Responde de manera justificada a la pregunta explicando el fenómeno de plasticidad sin generalizar

C. Responde de manera justificada y entre líneas se percibe el fenómeno de plasticidad pero no profundiza en él

C. Responde de manera justificada y entre líneas se percibe el fenómeno de plasticidad pero no profundiza en él

Jornadas de Formación e Innovación Docente del Profesorado | № 2 (2019) Esta obra se distribuye con la licencia Creative Commons Reconocimiento-NoComercial-SinObraDerivada Internacional (CC BY-NC-ND 4.0.) 


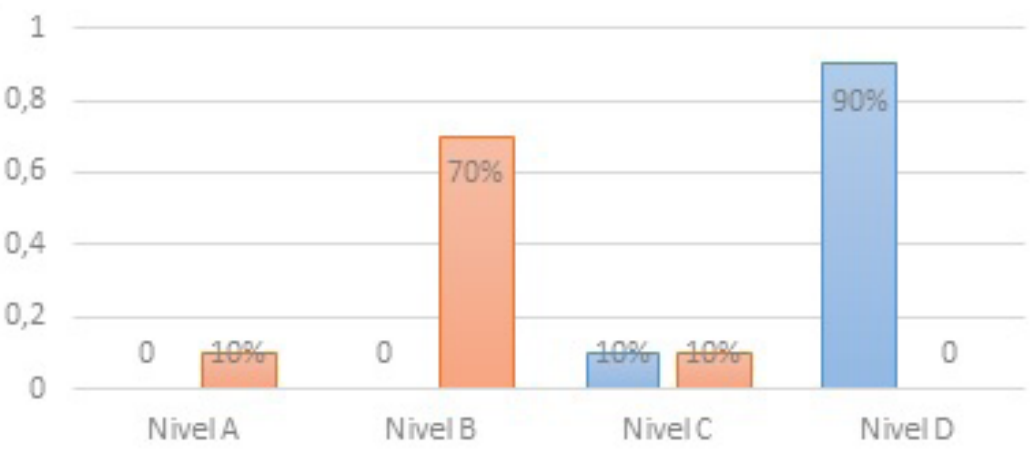

Cuestionario Inicial $\square$ Cuestionario Final

Gráfico 2. Escalera de niveles iniciales y finales a la pregunta ¿Se pueden entrenar nuestras capacidades? ¿A través de qué fenómeno se justifica?

En relación a la pregunta ¿Qué áreas conforman el desarrollo y qué tipo de intervenciones pueden realizarse para optimizarlas desde la Atención Temprana?, evidenciamos los niveles de su respuesta en la tabla 4 y en el gráfico 3, donde se muestra los porcentajes iniciales y finales en cada uno de los escalones de aprendizaje

Tabla 4. Niveles de respuesta de la pregunta ¿Qué áreas conforman el desarrollo y qué tipo de intervenciones pueden realizarse para optimizarlas desde la Atención Temprana?

A. Justifica y define las dimensiones que conforman el desarrollo del niño y esboza y planifica las intervenciones en cada una de las áreas

B. Define las dimensiones que conforman el desarrollo del niño y esboza y planifica las intervenciones en cada una de las áreas

C. Define las dimensiones que conforman el desarrollo y esboza las intervenciones en cada una de las áreas

D. No define las dimensiones y solo recoge algunos esbozos sin tener en cuenta todas las áreas de desarrollo

Jornadas de Formación e Innovación Docente del Profesorado | № 2 (2019) Esta obra se distribuye con la licencia Creative Commons Reconocimiento-NoComercial-SinObraDerivada Internacional (CC BY-NC-ND 4.0.) 


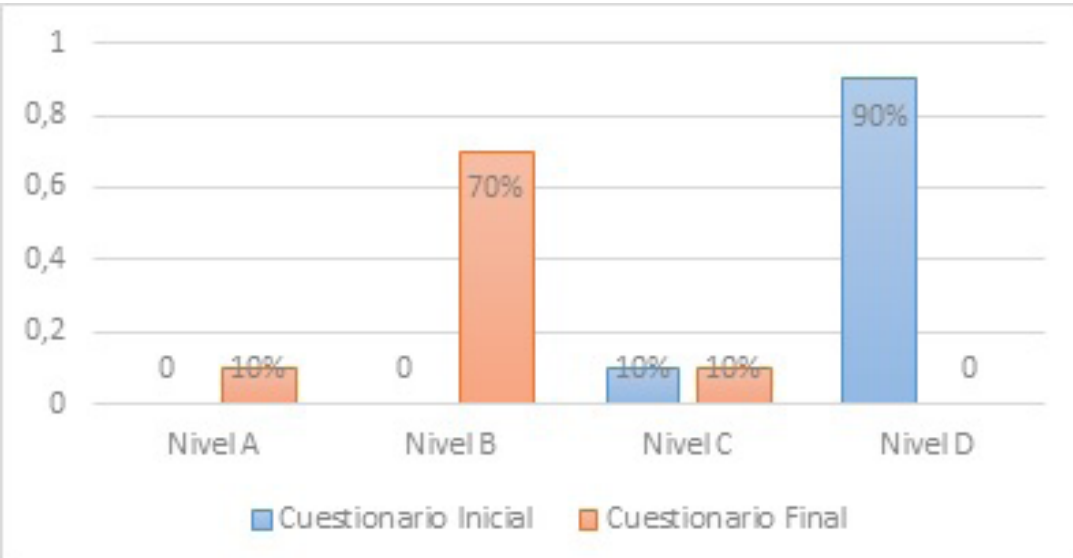

Gráfica 3. Escaleras de niveles iniciales y finales en la pregunta ¿Qué áreas conforman el desarrollo y qué tipo de intervenciones pueden realizarse para optimizarlas desde la Atención Temprana?

El análisis pormenorizado de las escaleras nos muestra que hay contenidos que no llegan a adquirirse en su máximo nivel, aunque si existe una mejora significativa en el proceso de aprendizaje. Un ejemplo de ello es la explicación del fenómeno de plasticidad neural. Podemos explicar su dificultad para alcanzar el nivel máximo de respuesta atendiendo a que es un concepto complejo para dominarlo en el segundo curso de la titulación. Para el próximo curso se intentará profundizar más en él y se preguntará de manera directa en el cuestionario, pues otra causa ha podido ser el tener que deducir que se preguntaba por este fenómeno y esa ambigüedad ha podido ser en parte responsable del resultado. Además del análisis exhaustivo de las escaleras de aprendizaje, las rúbricas diseñadas para cada actividad, las anotaciones en el diario de profesor y los datos extraídos de las actividades de gamificación, han servido de análisis en todo el proceso de enseñanza-aprendizaje de la asignatura. En la tabla 5 mostramos el ejemplo de la rúbrica diseñada para la evaluación de la actividad realizada en contexto real. 
Tabla 5. Rúbrica realizada para la actividad de AprendizajeServicio en las Escuelas Infantiles

\begin{tabular}{|c|c|c|c|}
\hline $\begin{array}{l}\text { Aspectos a } \\
\text { evaluar }\end{array}$ & $\begin{array}{l}\text { Nivel Inicial } \\
\text { (1 punto) }\end{array}$ & $\begin{array}{l}\text { Nivel intermedio } \\
\text { (2 puntos) }\end{array}$ & $\begin{array}{l}\text { Nivel Experto } \\
\text { (3 puntos) }\end{array}$ \\
\hline $\begin{array}{l}\text { Diseño de la } \\
\text { sesión }\end{array}$ & $\begin{array}{l}\text { El alumnado esboza } \\
\text { la tarea pero no de- } \\
\text { fine de manera acer- } \\
\text { tada ni los objetivos } \\
\text { ni los requisitos ne- } \\
\text { cesarios para llevarla } \\
\text { a cabo. No secuencia } \\
\text { las actividades ni pla- } \\
\text { nifica con sentido la } \\
\text { dinámica }\end{array}$ & $\begin{array}{l}\text { El alumnado diseña un } \\
\text { documento que pre- } \\
\text { senta los objetivos y } \\
\text { requisitos necesarios } \\
\text { para la dinámica ele- } \\
\text { gida pero no completa } \\
\text { la secuencia de activi- } \\
\text { dades y/o la planifica- } \\
\text { ción de la dinámica }\end{array}$ & $\begin{array}{l}\text { El alumnado diseña } \\
\text { una planificación ex- } \\
\text { celente, incluyendo los } \\
\text { objetivos que se pre- } \\
\text { tende, los recursos } \\
\text { necesarios para lle- } \\
\text { var a término la di- } \\
\text { námica, la secuencia } \\
\text { de actividades y la } \\
\text { temporalización }\end{array}$ \\
\hline $\begin{array}{l}\text { Material comple- } \\
\text { mentario al de- } \\
\text { sarrollo de la } \\
\text { dinámica }\end{array}$ & $\begin{array}{l}\text { El alumnado no pre- } \\
\text { senta ningún mate- } \\
\text { rial complementario } \\
\text { en su dinámica }\end{array}$ & $\begin{array}{l}\text { El alumnado presenta, } \\
\text { junto con el documento } \\
\text { de planificación, ma- } \\
\text { teriales específicos } \\
\text { diseñados para la acti- } \\
\text { vidad que se adecuan } \\
\text { a las necesidades de la } \\
\text { infancia }\end{array}$ & $\begin{array}{l}\text { Los materiales com- } \\
\text { plementarios diseña- } \\
\text { dos son creativos y } \\
\text { originales y el alum- } \\
\text { nado también se invo- } \\
\text { lucra hasta el punto de } \\
\text { llevar atrezos durante } \\
\text { su escenificación }\end{array}$ \\
\hline $\begin{array}{l}\text { Escenificación de } \\
\text { la dinámica con } \\
\text { los niños y niñas } \\
\text { de las escuelas } \\
\text { infantiles }\end{array}$ & $\begin{array}{l}\text { El alumnado se } \\
\text { muestra nervioso du- } \\
\text { rante la actividad y } \\
\text { no termina de mos- } \\
\text { trar su papel. No } \\
\text { interactúa con los ni- } \\
\text { ños y niñas y se frus- } \\
\text { tra en la tarea }\end{array}$ & $\begin{array}{l}\text { El alumnado defiende } \\
\text { su papel de manera } \\
\text { acertada aunque le } \\
\text { falta destreza y soltura } \\
\text { en el trato con los ni- } \\
\text { ños y niñas de las es- } \\
\text { cuelas infantiles }\end{array}$ & $\begin{array}{l}\text { El alumnado escenifica } \\
\text { su papel de manera } \\
\text { acertada, realizando su } \\
\text { actividad de manera } \\
\text { óptima y empatizando } \\
\text { con los más pequeños }\end{array}$ \\
\hline $\begin{array}{l}\text { Capacidad de } \\
\text { reflexión y } \\
\text { discusión }\end{array}$ & $\begin{array}{l}\text { Durante el desarrollo } \\
\text { de la actividad no se } \\
\text { encuentra una con- } \\
\text { cordancia entre lo re- } \\
\text { flejado en el proyecto } \\
\text { escrito y la puesta } \\
\text { en práctica de la } \\
\text { actividad }\end{array}$ & $\begin{array}{l}\text { El documento presen- } \\
\text { tado o la escenificación } \\
\text { de la dinámica contem- } \\
\text { pla concordancia y re- } \\
\text { fleja como objetivo } \\
\text { claro la optimización } \\
\text { del desarrollo y/o la } \\
\text { sensibilización en ma- } \\
\text { teria de diversidad } \\
\text { funcional }\end{array}$ & $\begin{array}{l}\text { Tanto el documento } \\
\text { presentado como la } \\
\text { escenificación de la } \\
\text { dinámica contempla } \\
\text { concordancia y refleja } \\
\text { como objetivo claro la } \\
\text { optimización del desa- } \\
\text { rrollo y/o la sensibili- } \\
\text { zación en materia de } \\
\text { diversidad funcional }\end{array}$ \\
\hline
\end{tabular}


Trabajo en equipo En la sesión de tra- El alumnado participa bajo en clase sobre la dinámica en las escuelas infantiles, el alumnado se muestra aislado y realiza su parte pero sin que se lleve a cabo un trabajo integrado y cooperativo en equipo y tanto el proyecto como la escenificación se esboza de forma integrada pero no se evidencia la cooperación de manera clara en el resultado final de la actividad
El alumnado participa en equipo de manera proactiva, coordinada, inclusiva, dinámica y participativa. Se observa en el resultado final una tarea con matiz cooperativo dónde todos han tenido un papel clave e imprescindible

Del mismo modo, otra innovación realizada este curso ha sido la incorporación de la IE en el aula, para lo que hemos utilizado el cuestionario en competencias emocionales de Ugarriza (2005), así como el cuestionario diseñado para evaluar la percepción del clima en el aula y todas las aportaciones de dinámicas recogidas en el manual específico de IE de Ortega (2017). Presentamos de manera resumida los descriptores iniciales y finales obtenidos en la percepción del clima de clase (tabla 6) y una gráfica que cumplimenta la significación de los hallazgos. Este cuestionario se presenta en escala tipo Likers, donde 1 es nada de acuerdo y 5 es totalmente de acuerdo.

Tabla 6. Descriptores iniciales y finales en el Cuestionario del Clima de clase

\begin{tabular}{|c|c|c|}
\hline Dimensiones del Clima de Clase & Valoración Inicial & Valoración Final \\
\hline Me siento feliz en la Facultad & $4,23(5-4)$ & $4,75(5-4)$ \\
\hline $\begin{array}{l}\text { Los compañeros nos ayudamos en la } \\
\text { Facultad }\end{array}$ & $4(5-3)$ & $4,62(5-3)$ \\
\hline $\begin{array}{l}\text { Siento que los Profesores ayudan en } \\
\text { la Facultad }\end{array}$ & $3,5(2-4)$ & $4,75(5-3)$ \\
\hline Me gusta mi Facultad & $4,25(5-4)$ & $4,62(5-4)$ \\
\hline Me gusta el ambiente de mi Facultad & $4,25(5-4)$ & $4,62(5-3)$ \\
\hline
\end{tabular}

Jornadas de Formación e Innovación Docente del Profesorado | № 2 (2019) Esta obra se distribuye con la licencia Creative Commons Reconocimiento-NoComercial-SinObraDerivada Internacional (CC BY-NC-ND 4.0.) 


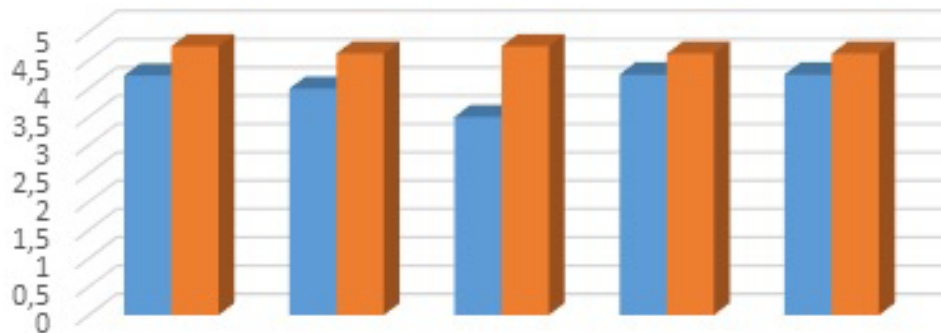

Me siento Los Siento que Megustami Megusta el

Felizen la compañeros los Facutad ambientede

Facutad nos profesores mifaculad

ayudamos ayudan en la

Facutad

Valoración Inicial Valoración Final

Gráfica 4. Valoración inicial y final del Clima del Aula

\section{Evaluación del Ciclo de mejora en el Aula:}

\section{Limitaciones y prospectiva para el futuro CIMA}

En todo el proceso de elaboración del CIMA, he tenido presente la experiencia acumulada en los ciclos anteriores $\mathrm{y}$, aunque creo que he llegado al modelo metodológico deseado, pienso que aún me falta madurez para conseguir un aprendizaje profundo en el alumnado y una excelente calidad docente. Además, tener la tarea trasversal de implantar la IE, ha podido alterar el tiempo dedicado a otras acciones, puesto que la reflexión tras la dinámica era tan rica que costaba acotar en minutos. Aunque este hecho ha podido ser una limitación, abordar esas competencias nos ha ayudado a estimular conductas proactivas en el alumnado que han redundado en su participación y motivación. En la gráfica 5 mostramos las actividades que ellos han resaltado como más significativas en su proceso de aprendizaje y en la gráfica 6 desglosamos la evaluación 
que el alumnado se otorga por su trabajo y dedicación a la materia, acompañada por la que le da a la asignatura y a la docente. Estos datos son extraídos de la encuesta específica desarrollada para ello, a la cual contestaron un total de 14 alumnos.

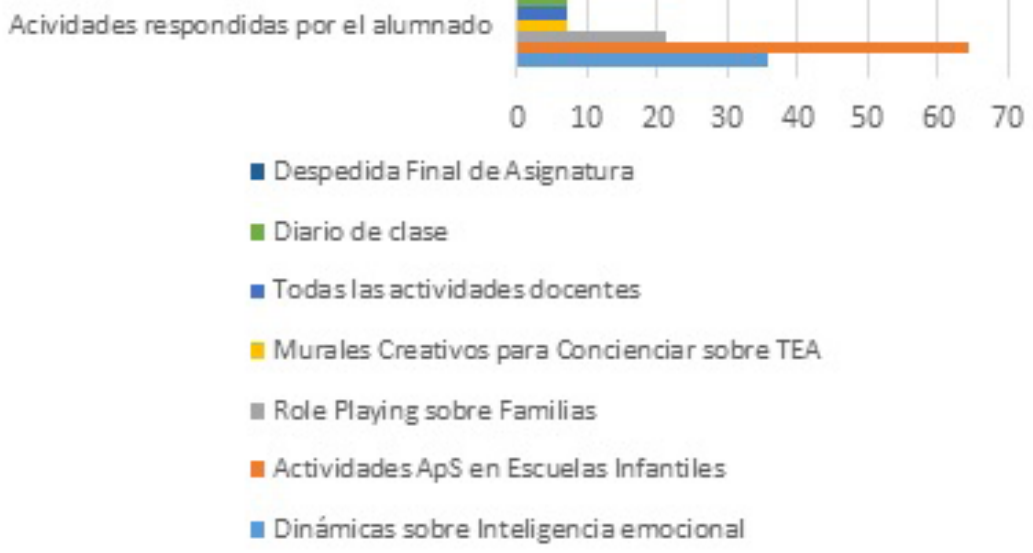

$\begin{array}{llllllll}0 & 10 & 20 & 30 & 40 & 50 & 60 & 70\end{array}$

- Despedida Final de A signatura

- Diario de clase

- Todas las actividades docentes

nurales Creativos para Concienciar sobre TEA

- Role Playing sobre Familias

- Actividades ApS en Escuel as Infantiles

- Dinámicas sobre Inteligencia emocional

Gráfica 5. Actividades que han tenido un aprendizaje significativo según la visión del alumnado

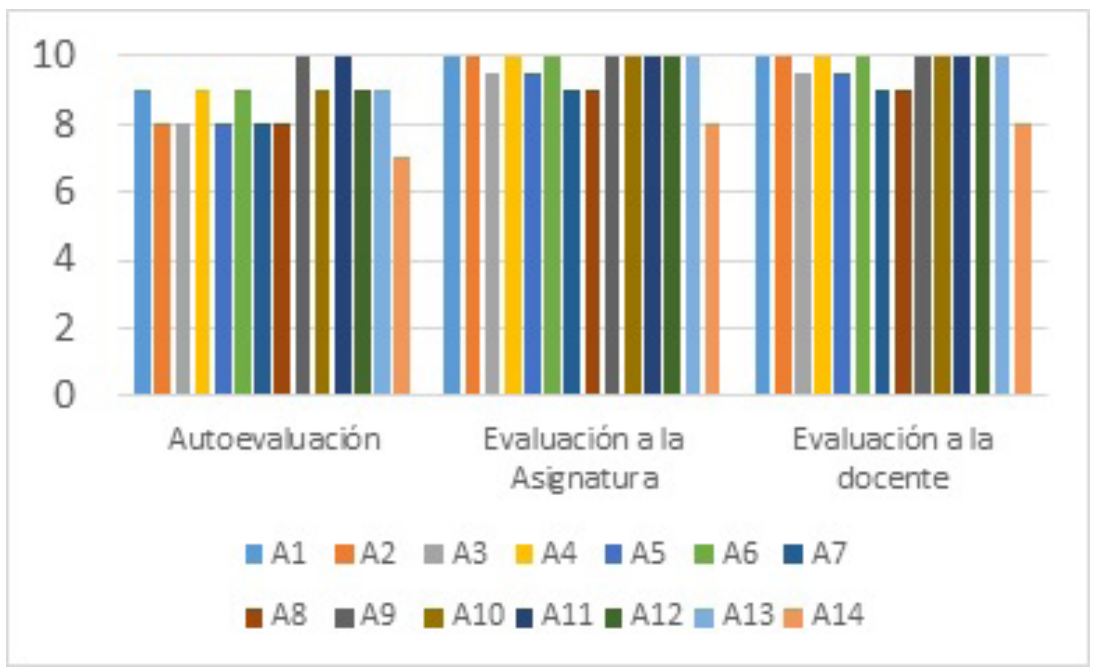

Gráfica 6. Autoevaluación, evaluación del docente y de la asignatura por parte del alumnado 
Aspectos de la experiencia para incorporar en la práctica docente habitual

En nuestra práctica docente pretendemos continuar con el diseño metodológico planteado, centrado en una filosofia de trabajo investigativa, donde la reflexión continuada y las ganas de mejorar sean piezas claves de nuestro propio proceso de aprendizaje docente. Por otro lado queremos seguir implementando la IE, puesto que el alumnado lo ha valorado como pertinente y como una herramienta que les ha ayudado a comprender la asignatura y el paradigma de la Atención Temprana. Seguiremos insistiendo en una docencia participativa y colaborativa, entendiendo que es la única manera de poder conseguir un aprendizaje profundo y esbozar el perfil del futuro profesional que la sociedad demanda, donde es importante el desarrollo de las inteligencias múltiples y donde deberemos hacer que el discente sea capaz de enamorarse de lo que aprende pues solo se puede aprender aquello que se ama (Mora, 2017). Por otro lado, nos parece interesante seguir indagando en nuevas metodologías docentes que resalten el papel que el alumnado puede tener en su propio proceso de aprendizaje, por ello queremos incluir metodologías creativas que respondan a las necesidades de las personas (ECO), así como implementar las rúbricas fluidas y otros instrumentos novedosos de evaluación de todos los protagonistas implicados en la docencia.

Principios didácticos que han guiado la experiencia y que deben permanecer en el futuro

Los principios que intentaremos mantener de manera fiel en nuestra docencia son los siguientes:

- La presentación de la asignatura irá precedida de un conocimiento de todos los discentes y de todo el profesorado, utilizando para ello la herramienta de la flor de tres pétalos: qué espero, qué conozco y qué me define. 
- Previo a cada unidad temática diseñaremos un cuestionario de conocimientos iniciales para poder adaptar las actividades al nivel de conocimiento de cada alumno y poder atender a la diversidad.

- Elaborar redes de contenidos y preguntas y organizar las secuencias de actividades de manera previa al desarrollo de la clase.

- Comenzar siempre con la formulación de una pregunta intrigante.

- Dar tiempo de trabajo al alumnado y potenciar su razonamiento crítico y clínico.

- Utilizar el diario del profesor para reflexionar de manera planificada.

- Proponer tareas y ejemplos que no solo contengan aspectos conceptuales y procedimentales sino que se enmarquen en aspectos actitudinales y en valores y que les ayude a ser futuros profesionales participativos, autónomos y críticos.

- Introducir formación relacionada con la IE, considerándola una pieza clave para empatizar con los pacientes y para ser profesionales excelentes.

- Intentar guiar el aprendizaje del alumnado, estando más tiempo en silencio y fomentando su interés e inquietud por descubrir nuevos hallazgos.

- Diversificar el tipo de actividades para que podamos llegar a la forma de aprender de cada discente.

- Acompañar todas las actividades con una hoja que contenga los procedimientos a seguir por escrito.

- Aplicar una evaluación continua dónde se tenga en cuenta el proceso, las tareas desarrolladas en las sesiones y el examen práctico y el teórico (incluyendo en este último tanto preguntas test como preguntas abiertas sobre casos clínicos).

- Establecer otros instrumentos de evaluación del profesorado, diferente al cuestionario de evaluación de la docencia.

- Incluir la autoevaluación del alumnado, a través de la evaluación entre iguales con el uso de la rúbrica. 


\section{Referencias Bibliográficas:}

Bain, K. (2004). Lo que hacen los mejores profesores universitarios. Valencia: Publicaciones Universidad de Valencia.

Benítez, M. (2018). Acciones innovadoras y reflexión docente en la asignatura "Fisioterapia en Atención Temprana" del Grado en Fisioterapia. Monográfico Jornadas de Formación e Innovación Docente del Profesorado 1, pp. 34-57. D.O.I.: http://dx.doi.org/10.12795/JDU.2018.i01.02

Finkel, D. (2008). Dar clases con la boca cerrada. Valencia: Publicaciones Universidad de Valencia.

Mora, F. (2017). Neuroeducación: Solo se puede aprender aquello que se ama. Madrid: Alianza Editorial.

Piñero, E. (2018). Implementación de ciclo de mejora docente en la asignatura Fisioterapia en Atención Temprana del Grado en Fisioterapia. Monográfico Jornadas de Formación e Innovación Docente del Profesorado 1, pp. 566-581. D.O.I.: http://dx.doi.org/10.12795/JDU.2018. 101.32

Porlán, R. (2017). Enseñanza Universitaria, Cómo mejorarla. Madrid: Editorial Morata.

Ortega-Cámara, A. (2017). Vivir en Inteligencia Emocional. Sevilla: Alegoría.

Ugarriza, N y Pajares, L. (2005). La evaluación de la Inteligencia Emocional a través del inventario de BarOn ICE: $N A$, en una muestra de niños y adolescentes. Persona $8,11-58$.

Jornadas de Formación e Innovación Docente del Profesorado | № 2 (2019) Esta obra se distribuye con la licencia Creative Commons Reconocimiento-NoComercial-SinObraDerivada 